\title{
Intracranial 4D Flow MRI: Toward Individualized Assessment of Arteriovenous Malformation Hemodynamics and Treatment-Induced Changes
}

\author{
S.A. Ansari, S. Schnell, T. Carroll, P. Vakil, M.C. Hurley, C. Wu, J. Carr, B.R. Bendok, H. Batjer, and M. Markl
}

\begin{abstract}
BACKGROUND AND PURPOSE: Arteriovenous malformations are an important etiology of hemorrhagic stroke. However, current imaging modalities and risk do not provide insights into individual AVM hemodynamics and its role in pathophysiology. The aims of this study are to determine whether intracranial 4D flow MR imaging can provide insights into arteriovenous malformation hemodynamics independent of the Spetzler-Martin grade and to report the changes in flow observed during staged embolization.
\end{abstract}

MATERIALS AND METHODS: Intracranial 3D blood flow was assessed in 20 patients with AVM (age $=39 \pm 15$ years, Spetzler-Martin grade ranging from 1-4) with the use of 4D flow MR imaging (temporal resolution $=45 \mathrm{~ms}$, spatial resolution $=[1.2-1.6 \mathrm{~mm}]^{3}$ ). AVM hemodynamics were visualized by means of time-integrated 3D pathlines depicting the AVM arterial feeding and venous draining patterns over the cardiac cycle. Analysis included the grading of feeding and draining velocities on a 3-point scale $(0=1 \mathrm{ow}<25 \mathrm{~cm} / \mathrm{s}, 1=$ medium $<50 \mathrm{~cm} / \mathrm{s}, 2=$ high $>50 \mathrm{~cm} / \mathrm{s}$ ). For 4 of 20 patients undergoing 4D flow MR imaging follow-up after staged embolization, peak velocities were quantified in arterial feeders, draining veins, the sagittal sinus, and contralateral arteries.

RESULTS: In $50 \%$ of the cases with Spetzler-Martin grade $>2$, heterogeneous flow (velocity grade differences $>1$ ) was found across arteries and veins. Velocities in draining veins increased from Spetzler-Martin grade $=1$ (grading $=0.5 \pm 0.6$ ) to Spetzler-Martin grade $\geq 3(1.1 \pm 0.6)$, whereas arterial velocities were similar $(1.7 \pm 0.6$ versus $1.5 \pm 0.6)$. In the postembolization subgroup of 4 patients, 4D flow MR imaging demonstrated successively more compact AVM and redistribution of velocities. Changes in arterial and venous velocities during treatment were highly different among individuals.

CONCLUSIONS: Spetzler-Martin grade does not reflect differences in 3D AVM arterial and venous hemodynamics, and an individual assessment of AVM hemodynamics may be needed for improved lesion characterization. Four-dimensional flow MR imaging may have the potential to monitor and guide embolization treatment planning.

ABBREVIATIONS: SMG = Spetzler-Martin grade; $\mathrm{PC}=$ phase-contrast, $\mathrm{FA}=$ feeding artery

A rteriovenous malformations are composed of a tangled network of feeding arteries directly connected to abnormal "arterialized" draining veins and are an important etiology of hemorrhagic stroke. ${ }^{1,2}$ The Spetzler-Martin grade, combining information

Received October 22, 2012; accepted after revision January 21, 2013.

From the Department of Radiology (S.A.A., S.S., T.C., P.V., M.C.H., J.C., B.R.B., M.M.) and Department of Neurological Surgery (S.A.A., M.C.H., B.R.B., H.B.), Feinberg School of Medicine; and Department of Biomedical Engineering (T.C., P.V., C.W., M.M.), McCormick School of Engineering, Northwestern University, Chicago, Illinois.

Grant support was provided by NIH-T32-EB005170, NIH-R01-HL088437, and NMH Excellence in Academic Medicine (EAM) Program, Advanced Cardiovascular MRI Research Center.

Please address correspondence to Michael Markl, PhD, Departments of Radiology and Biomedical Engineering, Northwestern University, 737 N Michigan Ave, Suite 1600, Chicago, IL 60611; e-mail: mmarkl@northwestern.edu

- Indicates open access to non-subscribers at www.ajnr.org

http://dx.doi.org/10.3174/ajnr.A3537 of AVM size, location, and venous drainage patterns, was originally proposed for AVM risk stratification. ${ }^{3}$ A high SMG, flow-induced aneurysms, and history of hemorrhagic rupture are widely accepted as major risk factors. However, these parameters represent empiric measures and do not provide insights into individual AVM hemodynamics and their role in pathophysiology and clinical presentations (eg, hemorrhage, ischemia, seizures). ${ }^{4,5}$ In addition, the predictive value of SMG is limited to the surgical morbidity of resection, and there is currently no risk score for AVM rupture itself.

Digital subtraction angiography combined with selective intra-arterial iodine contrast injections provides excellent AVM vascular imaging, angioarchitecture, and flow distribution. In addition, DSA guides AVM treatment via staged embolization procedures (endovascular superselective occlusion of AVM feeding arteries with nidal penetration). ${ }^{6,7}$ However, DSA procedures are associated with high cumulative contrast and radiation doses. 
Table 1: Demographics of 20 patients with AVM included in the study

\begin{tabular}{|c|c|c|c|c|c|c|c|c|}
\hline \multirow[b]{2}{*}{ AVM No. } & \multirow[b]{2}{*}{ Age, y } & \multirow[b]{2}{*}{ Sex } & \multirow[b]{2}{*}{ History } & \multirow{2}{*}{$\frac{\text { Venous Drainage }}{\text { Deep/Superficial }}$} & \multicolumn{2}{|c|}{ Location } & \multirow[b]{2}{*}{ AVM Size, cm } & \multirow[b]{2}{*}{ SMG } \\
\hline & & & & & Eloquent & Superficial/Deep & & \\
\hline $1^{\mathrm{a}}$ & 43 & $M$ & & $d+s$ & 0 & $\mathrm{~s}$ & $5.6 \times 4.1 \times 5.1$ & 3 \\
\hline 2 & 22 & M & Ruptured & $d+s$ & 0 & s & $2.5 \times 2.3 \times 2.4$ & 2 \\
\hline 3 & 68 & $\mathrm{~F}$ & & $d+s$ & 1 & $d$ & $4.0 \times 3.0 \times 2.5$ & 4 \\
\hline 4 & 44 & $M$ & & $d+s$ & 1 & $d$ & $1.0 \times 1.0 \times 1.0$ & 3 \\
\hline $5^{a}$ & 29 & $M$ & & $d+s$ & 0 & d & $4.0 \times 3.5 \times 3.4$ & 3 \\
\hline 6 & 48 & $\mathrm{~F}$ & & $\mathrm{~s}$ & 0 & s & $3.5 \times 3.5 \times 3.0$ & 2 \\
\hline 7 & 34 & M & Ruptured & s & 0 & s & $3.3 \times 2.3 \times 2.5$ & 1 \\
\hline 8 & 22 & $\mathrm{~F}$ & & s & 1 & s & $1.3 \times 1.6 \times 1.3$ & 2 \\
\hline 9 & 40 & $\mathrm{~F}$ & & $d+s$ & 0 & s & $3.0 \times 2.0 \times 4.0$ & 2 \\
\hline $10^{\mathrm{a}}$ & 43 & $M$ & Ruptured & $d+s$ & 1 & $\mathrm{~d}$ & $2.7 \times 3.1 \times 3.1$ & 3 \\
\hline 11 & 35 & $\mathrm{~F}$ & & $d+s$ & 0 & s & $3.1 \times 1.6 \times 1.0$ & 2 \\
\hline 12 & 25 & $\mathrm{~F}$ & & $d+s$ & 0 & s & $2.3 \times 2.3 \times 1.7$ & 2 \\
\hline $13^{a}$ & 21 & $\mathrm{~F}$ & & $d+s$ & 1 & s & $4.0 \times 3.4 \times 3.8$ & 4 \\
\hline 14 & 52 & $\mathrm{~F}$ & & s & 0 & s & $2.4 \times 2.1 \times 2.6$ & 1 \\
\hline 15 & 16 & $\mathrm{~F}$ & & s & 1 & $d$ & $2.3 \times 2.8 \times 2.8$ & 2 \\
\hline 16 & 66 & $M$ & & s & 0 & s & $0.8 \times 1.0 \times 0.8$ & 1 \\
\hline 17 & 49 & M & & s & 0 & s & $1.9 \times 2.4 \times 2.0$ & 1 \\
\hline 18 & 22 & $\mathrm{~F}$ & & s & 0 & s & $1.0 \times 2.5 \times 0.6$ & 1 \\
\hline 19 & 41 & $\mathrm{~F}$ & & $d+s$ & 1 & s & $6.6 \times 3.7 \times 5.2$ & 4 \\
\hline 20 & 55 & M & & s & 0 & s & $2.1 \times 3.6 \times 2.6$ & 2 \\
\hline
\end{tabular}

a Patients for whom follow-up 4D flow MRI was obtained during staged embolization. Note:-d indicates deep; s, superficial.

Moreover, noninvasive monitoring of embolization-induced changes has been limited by the lack of modalities to quantitatively assess AVM hemodynamics.

A number of studies reported the use of noninvasive 4D (3D + time) MR angiography for the evaluation of the MR contrast agent dynamics in the cerebral vasculature and AVM. ${ }^{8-13}$ More recently, 4D flow MR imaging, which adds a new dimension (quantitative 3-directional blood flow velocities) has been used for the in vivo measurement and visualization of intracranial arterial and venous 3D blood flow ${ }^{14-18}$ and to assess complex 3D flow patterns in AVMs in a small number of cases. ${ }^{19,20}$ It was the aim of this study to apply 4D flow MR imaging in patients with AVM to assess AVM 3D hemodynamics and to monitor changes during treatment by staged embolization. We hypothesize that intracranial $4 \mathrm{D}$ flow MR imaging can provide further insights into AVM hemodynamics independent of SMG and can identify changes in arterial feeding and venous draining blood flow velocities during staged embolization.

\section{MATERIALS AND METHODS Study Population}

Intracranial 4D flow MR imaging was obtained in 20 patients with AVM (age $=39 \pm 15$ years, 11 female). Patient demographics and AVM characteristics are outlined in Table 1 . The patient cohort included 3 ruptured and 17 unruptured AVMs with SpetzlerMartin grades ranging from 1-4. DSA confirmed deep AVM location in 5 of 20 patients and deep venous drainage in 11 of 20 patients. In 4 of 20 patients with AVM, follow-up 4D flow MRI was obtained during interventional treatment by staged embolization. For all patients included in this study, liquid Onyx (ethylene-vinyl alcohol copolymer; ev3, Irvine, California) was used as embolic material. The study was carried out in accordance with an institutional review board protocol, which permitted retrospective chart review.

\section{MR Imaging}

All measurements were performed with the use of $1.5 \mathrm{~T}$ and $3 \mathrm{~T}$ MR systems (Avanto \& Trio; Siemens, Erlangen, Germany), with a standard 12-channel head coil. MR imaging included time-offlight MR angiography and 3D T1-weighted MPRAGE for the localization of the AVM. Cerebral 3D blood flow was measured by using electrocardiographic gated and time-resolved (cine) 3D phase-contrast (PC) MR imaging with 3-directional velocity encoding (also termed "4D flow MR imaging"). ${ }^{15,21}$ Data were acquired in an axial oblique 3D volume covering the AVM. Pulse sequence parameters for $4 \mathrm{D}$ flow $\mathrm{MR}$ imaging were as follows: velocity sensitivity $=100-120 \mathrm{~cm} / \mathrm{s}$, flip angle $=15^{\circ}$, temporal resolution $=45 \mathrm{~ms}$, section thickness $=1.2-1.6 \mathrm{~mm}$, slab thickness $=52.8-70.4 \mathrm{~mm}$, spatial resolution $=(1.2-1.6)^{3} \mathrm{~mm}^{3}$. Total acquisition times for $4 \mathrm{D}$ flow MR imaging were heart rate-dependent and ranged between 15-20 minutes.

\section{Data Analysis}

All 4D flow data underwent preprocessing to correct for Maxwell terms, eddy currents, and velocity aliasing with the use of inhouse software programmed in Matlab (MathWorks, Natick, Massachusetts), as described previously. ${ }^{22}$ In addition, a 3D phase-contrast MR angiogram was calculated from the 4D flow data. ${ }^{23,24}$ Anatomy, blood flow, and vascular geometry included in the resulting data were then further analyzed by means of a 3D visualization software package (EnSight; CEI, Apex, North Carolina). Three-dimensional blood flow visualization was based on the calculation of time-resolved 3D pathlines to depict the temporal evolution of blood flow in intracranial vessels. Three-dimensional pathlines were emitted within the entire intracranial vasculature, that is, all arteries and veins depicted by the 3D PCMRA data that defined the vascular lumen; 15,000-25,000 emitter points were equally distributed within the 3D-PC-MRA vessel lumen and used to initiate the calculation of time-resolved $3 \mathrm{D}$ 

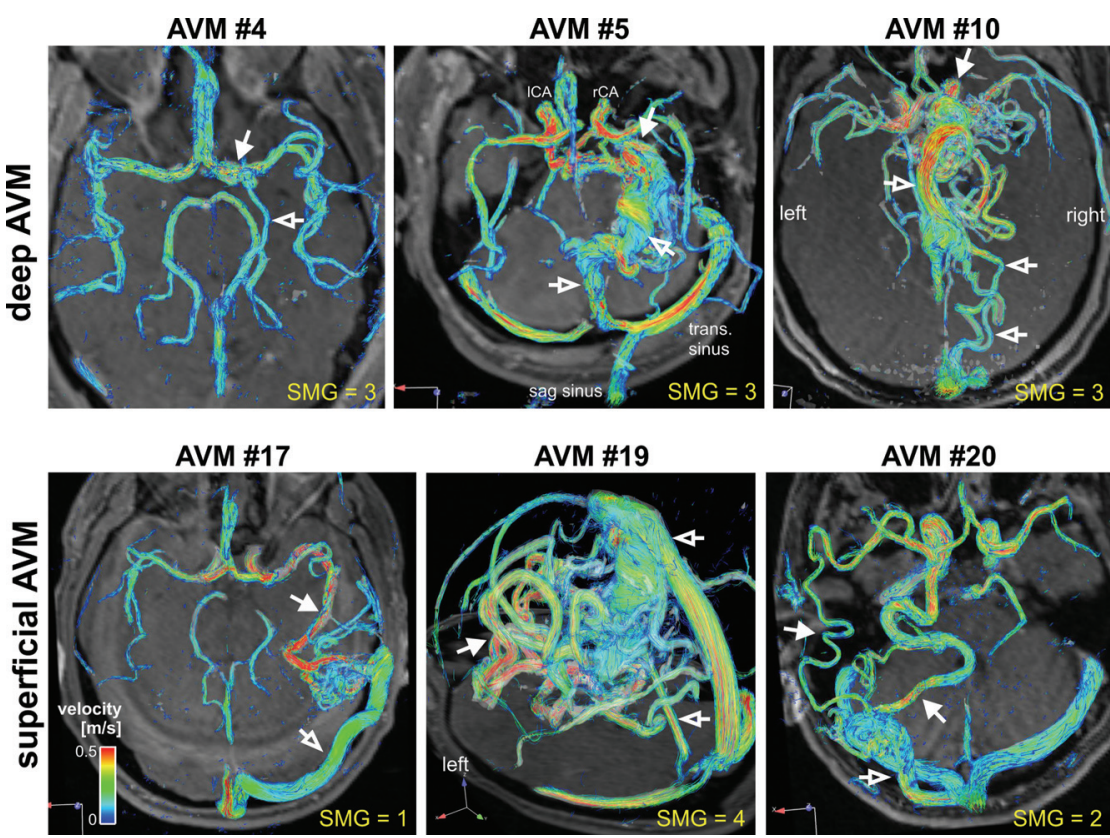

FIG 1. AVM hemodynamics visualized by time-integrated 3D pathlines in 6 of 20 patients. Colorcoding shows the local velocity of blood traveling along the traces during 1 cardiac cycle. Different AVM sizes as well as feeding artery (solid white arrows) and draining vein patterns (white open arrows) can clearly be appreciated. AVM 4, 5, and 10: Deep AVM location and deep venous drainage resulting in with high SMG. AVM 17, 19, and 20: Superficial AVM location with superficial and deep venous drainage.

pathlines. Time-integrated pathlines were derived from the timeresolved pathlines by depicting the collective pathline traces over 1 cardiac cycle, that is, the cumulative path of blood flow over 1 cardiac cycle (Figs 1-3).

In contrast to alternative visualization options such as streamlines (traces along the 3D velocity field at 1 instance in time) or vector graphs (depiction of the $2 \mathrm{D}$ velocity field at a selected location and 1 point in time), time-intergraded $3 \mathrm{D}$ pathlines were chosen to provide an overview over the spatial and temporal distribution of 3D blood flow within a single image. In addition, pathline emission from the entire intracranial vasculature provided by the 3D PC-MRA data provided the advantage of visualizing $3 \mathrm{D}$ blood flow in all $\mathrm{MR}$ visible arteries and veins inside the entire 3D data volume.

The color coding of the time-integrated traces reflects the velocities of blood traveling along the pathlines during 1 cardiac cycle and was used to estimate the range and magnitude of blood flow velocities with the cardiac cycle. Previous studies have used similar approaches to analyze 3D blood flow distributions in patients with high-risk aortic plaques. ${ }^{25}$ In consensus reading, 2 observers identified the number of arterial feeders and draining veins visible in the $4 \mathrm{D}$ flow data. Feeding/draining velocities in each vessel were visually graded as 0 (low flow $<25 \mathrm{~cm} / \mathrm{s}$ ), 1 (medium flow $<50 \mathrm{~cm} / \mathrm{s}$ ), and 2 (high flow $>50 \mathrm{~cm} / \mathrm{s}$ ). The grading relied on the identification of clearly visible regions within individual arteries with velocity $<25 \mathrm{~cm} / \mathrm{s}$ or $>50 \mathrm{~cm} / \mathrm{s}$. AVM velocity distribution was labeled as heterogeneous if velocity grades varied across vessels. For patients with follow-up 4D flow MR imaging after embolization, peak velocities were quantified in arterial feeders, draining veins, the sagittal sinus, and contralateral arteries. In addition, diameters of arterial feeders were measured and classified as homogeneous (maximum vessel diameter differences sinuses (Table 2).
$<20 \%$ ) or heterogeneous (maximum vessel diameter differences $>20 \%$ ).

Time needed for data analysis included approximately 10-15 minutes for preprocessing, 40-60 minutes for $3 \mathrm{D}$ blood flow visualization by use of time-integrated pathlines, and 15-20 minutes for placement of analysis planes and flow quantification.

\section{Three-Dimensional Flow Connectivity Mapping}

In a subset of 2 patients with $\mathrm{SMG}=4$, large and highly complex AVMs, 3D pathlines that originated from emitter planes in individual feeding arteries or draining veins were used to visualize different flow pathways inside the AVM. Traces emitted from each of the emitter planes were color-coded according to their anatomic origin to identify the blood flow paths with respect to their vascular source (3D flow connectivity mapping), as described previously. ${ }^{26}$

\section{RESULTS}

Twenty-seven 4D flow MR imaging scans were performed in the 20 patients. Four patients who were undergoing treatment with staged embolization received additional follow-up 4D flow MRI after embolization procedures: 2 follow-up examinations in 3 patients (AVM 1, 5, and 13) and 1 follow-up examination in 1 patient (AVM 10). Three-dimensional visualization of AVM hemodynamics was successfully performed in 18 of 20 patients. Two patients were excluded from the analysis because of motion artifacts (AVM 11) or small AVM size $<1 \mathrm{~cm}$ (AVM 16). In all remaining cases, the 4D flow MR imaging volume encompassed the entire AVM including the arterial supply and the venous drainage.

\section{Three-Dimensional AVM Hemodynamics}

Time-integrated pathlines in Fig 1 illustrate the complex distribution of blood flow inside the AVM and surrounding vessels for 6 patients with different AVM sizes, location, and draining patterns. Dominant arterial feeders and large deep and superficial draining veins were clearly identified in all cases. Note that blood flow characteristics, presence of high velocities (yellow/red colors) and distribution of velocities in AVM feeding arteries and draining veins were highly variable despite identical SMG. Particularly, venous blood flow velocities were substantially different and often unusually high $(>30-50 \mathrm{~cm} / \mathrm{s})$ compared with normal venous flow $(15-25 \mathrm{~cm} / \mathrm{s})^{27}$ in the sagittal and transverse

Visual grading of time-integrated 3D particle traces confirmed considerable heterogeneity in $3 \mathrm{D}$ arterial feeding and venous draining patterns even for patients with identical SMG (Table 2). The number of feeding arteries (1-4), draining veins (1-3), and velocity distribution was highly variable. In $50 \%$ of the cases with 
Table 2: Summary of the results of visual flow pattern grading in patients with AVM $(n=18)$

\begin{tabular}{|c|c|c|c|}
\hline & SMG $1(n=4)$ & SMG $2(n=7)$ & SMG $\geq 3(n=7)$ \\
\hline \multicolumn{4}{|l|}{ Arterial feeders } \\
\hline No. of arteries & Single & Single and multiple (1-3) & Single and multiple (1-4) \\
\hline Flow across arteries & Homogeneous & Heterogeneous $n=4$ & Heterogeneous $n=3$ \\
\hline \multirow[t]{3}{*}{ Velocity grading } & $1.7 \pm 0.6$ & $1.4 \pm 0.5$ & $1.5 \pm 0.6$ \\
\hline & Range $=1-2$ & Range $=1-2$ & Range $=0-2$ \\
\hline & Median $=2$ & Median $=1$ & Median $=1$ \\
\hline \multicolumn{4}{|l|}{ Venous drainage } \\
\hline No. of veins & $\begin{array}{l}\text { Superficial only } \\
\quad(1-2)\end{array}$ & $\begin{array}{l}\text { Superficial/deep } \\
\quad(1-3)\end{array}$ & $\begin{array}{l}\text { Superficial/deep } \\
\quad(1-3)\end{array}$ \\
\hline Flow across veins & $\begin{array}{l}\text { Heterogeneous } \\
\quad n=2\end{array}$ & $\begin{array}{l}\text { Heterogeneous } \\
\qquad \begin{array}{l}n=3\end{array}\end{array}$ & $\begin{array}{l}\text { Heterogeneous } \\
\quad n=4\end{array}$ \\
\hline \multirow[t]{3}{*}{ Velocity grading } & $0.5 \pm 0.6$ & $0.8 \pm 0.8$ & $1.1 \pm 0.6$ \\
\hline & Range $=0-1$ & Range $=0-2$ & Range $=0-2$ \\
\hline & Median $=0.5$ & Median = 1 & Median = 1 \\
\hline
\end{tabular}
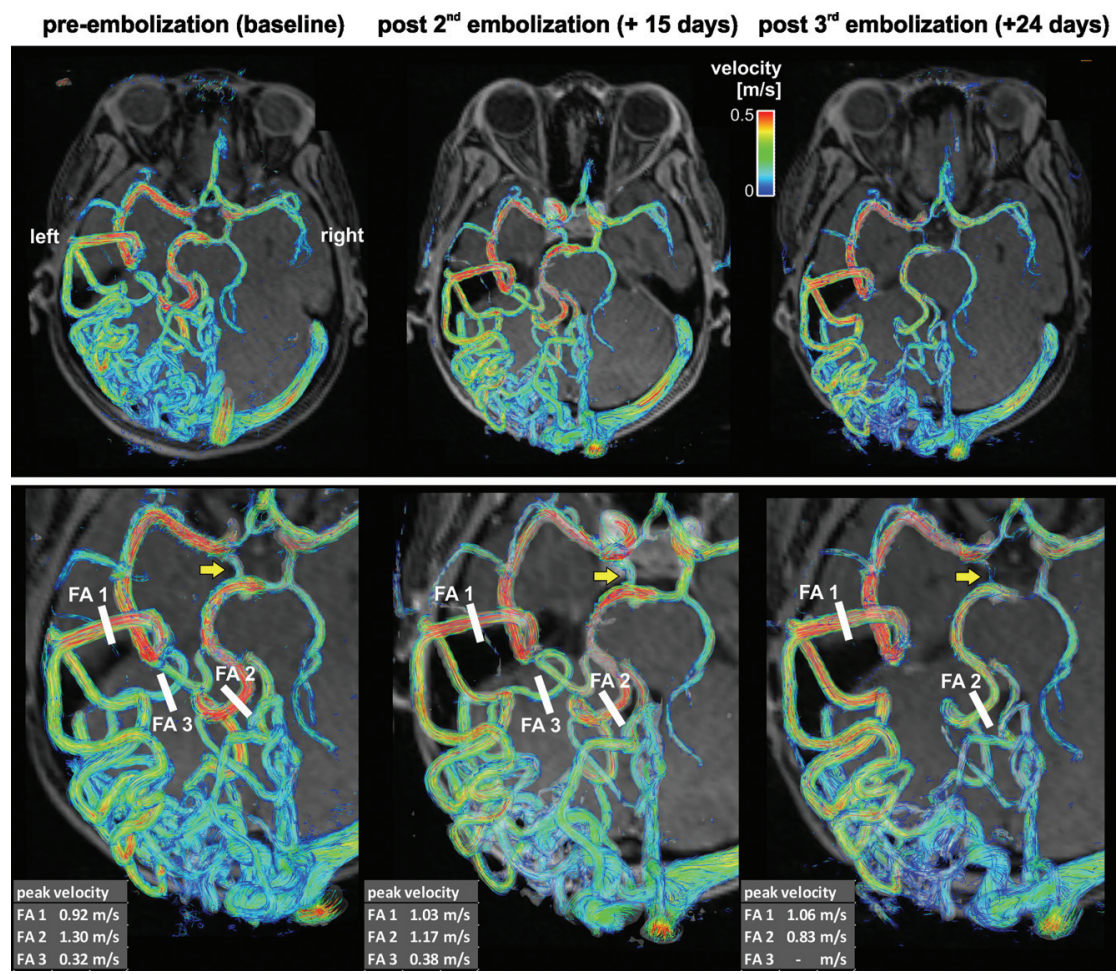

FIG 2. Three-dimensional blood flow visualization and quantification in a patient with a large unruptured temporo-occipital AVM (AVM 13, SMG =4). Complex arterial feeding and convoluted hemodynamics as well as differences in pre-embolization and postembolization vascularization and hemodynamics are clearly visible. Flow quantification in 3 feeding arteries (FA 1-3) revealed substantial changes and redistribution of peak velocities during the course of staged embolization. Note that FA 3 was embolized during the third procedure. Note that embolization resulted in reduced flow in the right posterior communicating artery (yellow arrows). As evident from the changes in peak velocity in feeding arteries FAl and FA2, the final embolization stage resulted in a substantial decrease in peak velocity in the FA2 systems. As a result, shunting to the FA2 system through the right posterior communicating artery was reduced, which resulted in its diminished appearance.

$>1$ feeding artery $(S M G \geq 2)$ or draining vein $(S M G \geq 1)$ heterogeneous flow was found, whereas the remaining patients showed homogeneous flow across arteries and veins. Overall, increased SMG corresponded to higher venous velocities (velocity grading increased by $120 \%$ from SMG $=1$ to $S M G \geq 3$ ), whereas arterial velocities were similar (decrease by $13 \%$ from $S M G=1$ to $S M G$ 23). Interestingly, for the 7 AVMs with heterogeneous velocity distribution across arterial feeders, only 2 showed a heterogeneous distribution of vessel diameters.

\section{Follow-Up During Staged Embolization}

As has been shown previously, ${ }^{28}$ the embolization material (Onyx) appeared to be hypointense on MR images, did not interfere with MR images, and we did not observe artifacts related to embolic material in our study cohort.

As illustrated for a large temporo-occipital AVM (AVM 13, SMG = 4) in Fig 2, multiple embolization procedures resulted in a more compact AVM and altered velocity distribution as visualized by a reduced nidal network and changes in color-coding (reduction in velocities). Peak velocities in the 3 main feeding arteries (FA 1-3) underwent redistribution during treatment. Initially, the highest peak velocity in feeding artery FA 2 was reduced during the course of staged embolization. Moreover, the peak velocity ratio in FA 1 versus FA 2 (pre-embolization: FA 2 40\% higher compared with FA 1) was inverted after the third embolization procedure $(28 \%$ higher in FA 1 compared with FA 2).

Similar results (successively more compact AVM, redistribution of velocities) were found in all 4 cases undergoing staged embolization. Quantitative analysis (Fig 3B) revealed marked alterations during treatment in peak velocities in all vascular territories. Changes in velocities also occurred in locations distant to the site of arterial embolization such as the sagittal sinus and contralateral arteries (large artery in hemisphere not affected by the AVM).

\section{Three-Dimensional Flow Connectivity Mapping}

Figure 4 demonstrates the use of flow connectivity mapping in 2 large AVMs. In comparison, velocity color-coded time-integrated pathlines for the same AVMs as shown in Fig 1 (AVM 19) and Fig 2 (AVM 13) provide an overview of AVM vascularization and velocity distribution. However, individual feeding and draining pathways are difficult to identify because of the complexity of the lesion. In these cases, flow connectivity mapping mimicking superselective iodine contrast injections provides a noninvasive method to selectively identify and delineate compartmentalized AVM feeding and draining pathways.

\section{DISCUSSION}

The findings of this study demonstrate the potential of $4 \mathrm{D}$ flow MR imaging for the 3D visualization of complex flow patterns 


\section{A 3D flow visualization}
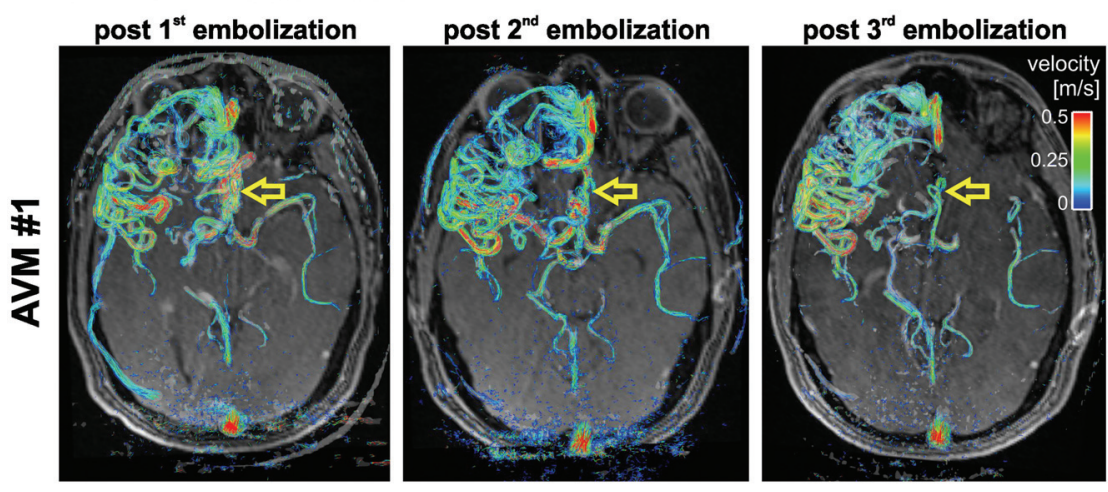

\section{$B$ Flow velocity quantification}
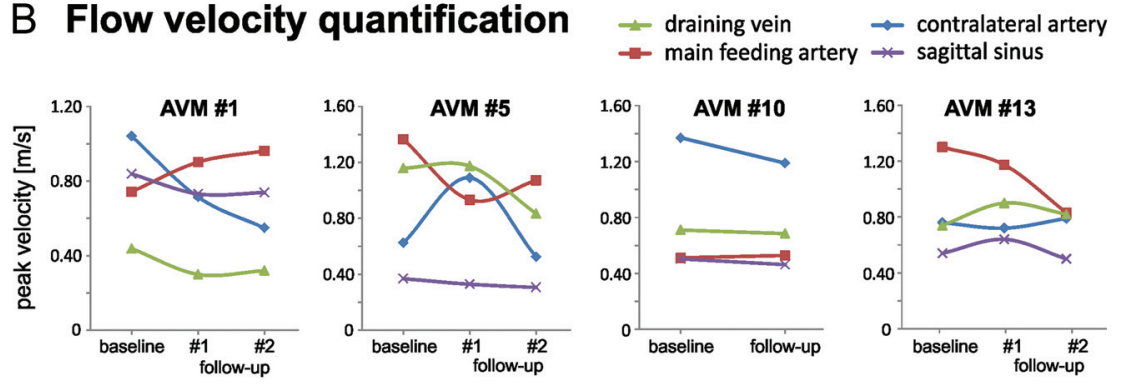

FIG 3. A, Three-dimensional blood flow in a large frontal AVM (AVM I, SMG = 3). Staged embolization resulted in compaction of the AVM with reduced blood flow velocities (yellow arrows). B, Quantification of postinterventional changes in AVM hemodynamics on the basis of $4 \mathrm{D}$ flow MR imaging after multiple embolization procedures in all 4 patients who underwent MR imaging follow-up. the risk of hemorrhage postembolization, which is estimated at approximately $3-5 \% .^{33-36}$

In the small cohort of patients included in our study, 4D flow MR imaging revealed intricate vascularization and heterogeneous flow patterns of AVMs independent of size, location, and severity of grade as specified by the SMG classification system. Different patterns of venous drainage with high variability in regional blood flow velocities indicate the complex nature of AVMs and the need for individual evaluation of flow characteristics. For example, in AVMs with heterogeneous peak velocity distribution across arterial feeders, only a small fraction showed differences in vessel diameters. This finding indicates that AVM hemodynamic parameters are not simply determined by vessel size but show a complex behavior that can in general not be directly linked to simple geometric markers.

The results of this study confirm the limited value of SMG for AVM risk stratification beyond the surgical morbidity of resection. New quantitative markers of AVM hemodynamics such as within AVMs and its interconnections with the surrounding arterial and venous vascular system. Despite similar SMG, AVMs demonstrated heterogeneous and divergent arterial/ venous patterns and flow velocity distributions, indicating the potential of in vivo $3 \mathrm{D}$ flow measurements to further characterize AVMs beyond the traditional empiric measures used for SMG.

In a subgroup of patients, 4D flow MR imaging was used for AVM treatment monitoring during multiple staged embolizations. Marked changes in velocities in all territories including sagittal sinus and contralateral arteries during follow-up demonstrate the potential of 4D flow MR imaging to evaluate the systemic impact of embolization on cerebral flow in multiple vascular territories.

Intracranial AVMs are highly complex and diverse lesions. Each AVM can consist of several types of tissues, compact or diffuse clusters of true nidal arteriovenous vessels, and areas of direct arteriovenous fistula in which a large arterial channel directly drains into the venous system. ${ }^{29}$ Current preoperative noninvasive imaging methods often require assessment with invasive DSA and even superselective intra-arterial injections before embolization. ${ }^{30}$ DSA is also routinely performed in the preoperative evaluation to more accurately map the anatomy or angioarchitecture of the lesion (feeding arteries, nidus, draining veins, venous outflow/stenoses, and flow-induced aneurysms) but provides only rudimentary information on flow dynamics. ${ }^{31,32}$ However, the impact of altered feeding patterns on the hemodynamics in AVMs with complex vascularization and flow patterns is difficult to predict even if feeding arteries can be clearly identified. Incomplete information on altered AVM hemodynamics may contribute to distribution of peak velocities across feeders or changes in feeding and draining velocities during treatment may thus have the potential to provide contributing factors to new and improved risk assessment for AVMs. Additional information that could be obtained during the same MR imaging examination, such as cerebral microvascular perfusion (cerebral blood flow and volume), could be incorporated into the hemodynamics analysis to offer a better multi-parametric functional assessment of AVMs. In this context, a detailed assessment of AVM blood flow distribution by 4D flow MR imaging in combination with parameters of microvascular perfusion may help to better understand the often controversial topic of vascular steal associated with AVMs. Four-dimensional flow MR imaging may offer the opportunity to study these effects and the impact of therapy and intervention (eg, reversal of hypoperfusion by removal of shunting) on vascular steal. Future studies in larger cohorts and long-term follow-up are needed to evaluate the diagnostic value of such parameter combinations as potential future risk scores for AVM characterization.

A previous $4 \mathrm{D}$ flow study by Hope et $\mathrm{al}^{19}$ reported findings in 1 patient with a large left frontoparietal AVM compared with 2 healthy volunteers. Similar to our findings, the AVM patient showed increased venous flow and high flow in arterial feeders (3.3 times faster flow was noted in the 3 largest arterial feeders supplying the AVM via the ipsilateral internal carotid artery in comparison to the healthy controls).

In a recent study, Chang et $\mathrm{al}^{20}$ investigated 10 patients with AVM with 4D flow MR imaging to primarily assess wall shear stress in AVM feeding and contralateral normal arteries. Patients were divided into 2 subgroups, with the first group harboring 


\section{AVM \#13}
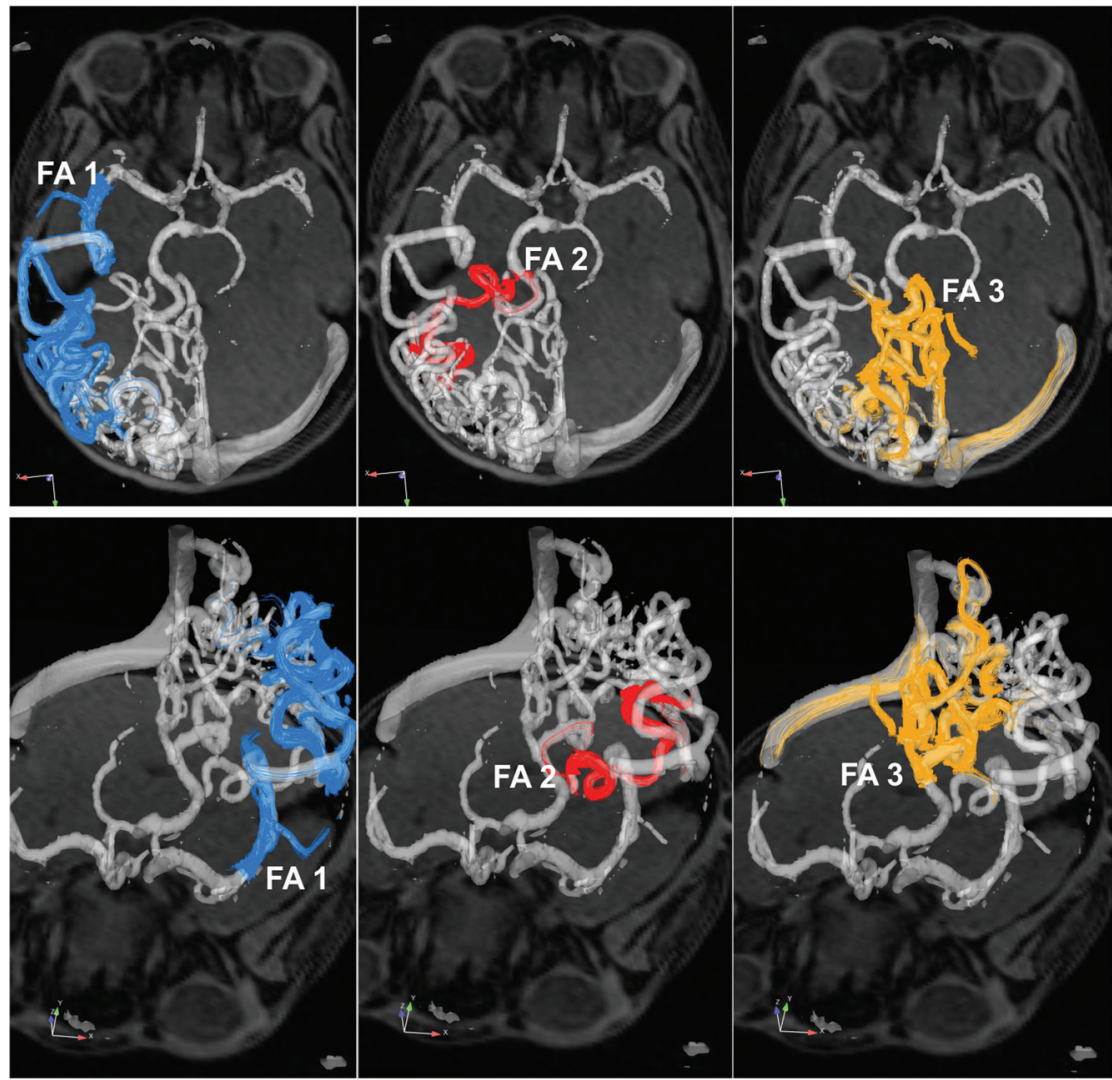

\section{AVM \#19}

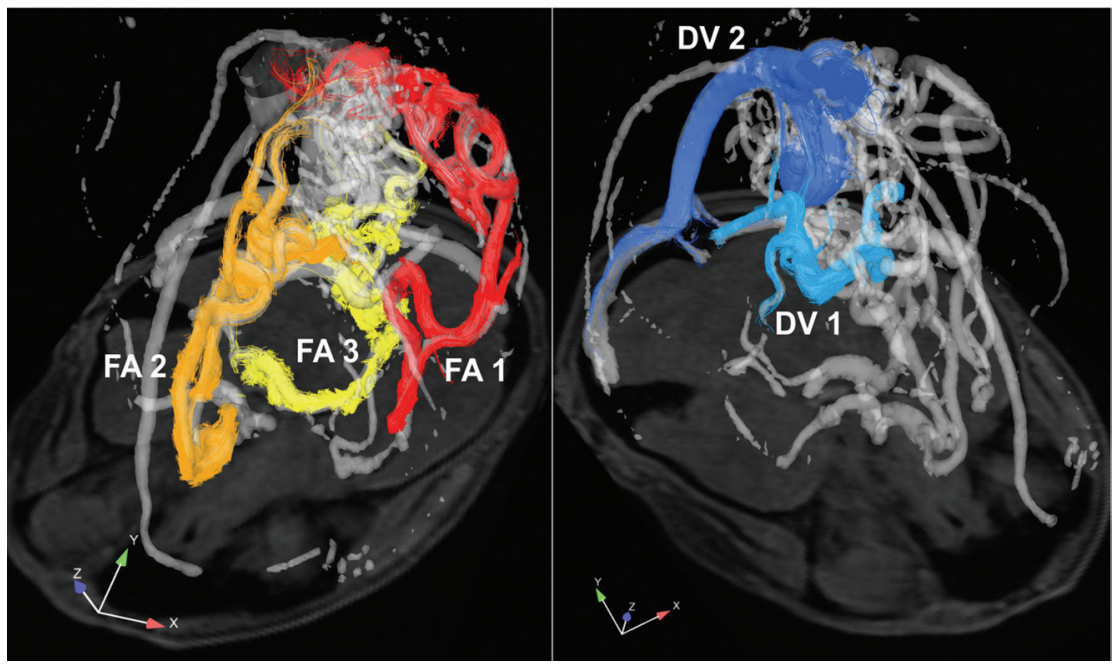

FIG 4. Flow connectivity mapping of AVM feeding and draining patterns in 2 large AVMs with complex vascularization (both SMG $=4$ ). The gray shaded iso-surfaces show 3D-PC-MRA that was calculated from the 4D flow MR data. FA = feeding artery; DV = draining vein.

asymptomatic AVMs of SMG $=3$ and the second group with ruptured AVMs of SMG 1-4, presenting with hemorrhage and neurologic deficits. In the first group, wall shear stress was similar for AVM feeding and contralateral normal arteries, as opposed to group 2, which exhibited a significant decrease of wall shear stress in normal vessels. An increased time-averaged velocity and flow in the feeding arteries compared with the contralateral arteries was found in both groups.
To our knowledge, these studies constitute the only reports of the application of 4D flow MR imaging to AVMs to date. Our study investigated a larger cohort of patients with AVM and clearly demonstrated the ability of 4D flow MR imaging to assess qualitative and quantitative changes in AVM hemodynamics during staged embolization. We were able to identify highly variable alterations in arterial or venous flow velocities that could be caused by compensatory flow after parallel feeder occlusion, aggressive intranidal embolization, or venous outflow penetration or obstruction. In this context, 4D flow MR imaging may identify subtleties and nuances of AVM angioarchitecture that supplement other modalities such as DSA. As such, the technique may offer a new tool for pre-interventional and postinterventional monitoring of intracranial hemodynamics and provide additional information on regional blood flow velocities and flow patterns. A particular advantage of the presented technique is related to the fact that imaging can easily and noninvasively be performed at multiple instances in conjunction with routine MR imaging without the need for ionizing radiation.

\section{Limitations}

A major limitation of our study is the small number of patients investigated. Although this is the largest series to date studying AVMs with 4D flow MR imaging for specific flow patterns, it remains a feasibility study and the conclusions are limited with respect to the clinical utility of the findings. Correlation of hemodynamic findings with either risk stratification or patient outcomes is unclear, possibly because of heterogeneous flow architecture of AVMs, inadequate power, and the paucity of ruptured AVMs. Another limitation is the limited spatial resolution resulting from the need to cover the entire intracranial vasculature for the AVM investigation, which also must be acquired in a reasonable scan time. These technical issues may be solved with advanced acceleration techniques and a dual-velocity encoding approach in future investigations. Further studies are warranted, including serial $4 \mathrm{D}$ flow imaging to evaluate its potential for improving interventional planning and postinterventional monitoring. 


\section{CONCLUSIONS}

Four-dimensional flow MR imaging provides the opportunity for noninvasive assessment and visualization of complex feeding and draining patterns in cerebral AVMs. Phase-contrast MRA techniques allow the qualitative and quantitative evaluation of AVM hemodynamics and may offer the potential for risk stratification, more precise monitoring during staged embolization, and/or improved treatment planning.

Disclosures: Timothy Carroll—RELATED: Grant: NIH/NHLBI;* UNRELATED: Grants/ Grants Pending: NIH/NIBIB; Patents (planned, pending or issued): Several, unrelated to work. Parmede Vakil—RELATED: Grant: NIH;* UNRELATED: Grants/Grants Pending: NIH. * James Carr-UNRELATED: Grant/Grants Pending: Siemens, ${ }^{*}$ Comments: Research support. Michael Markl—UNRELATED: Grant: NIH, ${ }^{*}$ Comments: NIH-T32EB005170, NIH-R01-HL088437 ( ${ }^{*}$ money paid to institution).

\section{REFERENCES}

1. Rigamonti D, Hadley MN, Drayer BP, et al. Cerebral cavernous malformations: incidence and familial occurrence. $N$ Engl J Med 1988;319:343-47

2. Arteriovenous malformations of the brain in adults. $N$ Engl J Med 1999;340:1812-18

3. Spetzler RF, Martin NA. A proposed grading system for arteriovenous malformations. J Neurosurg 1986;65:476-83

4. Batjer HH, Devous MD Sr, Seibert GB, et al. Intracranial arteriovenous malformation: relationships between clinical and radiographic factors and ipsilateral steal severity. Neurosurgery 1988;23:322-28

5. Stapf C, Mast H, Sciacca RR, et al. Predictors of hemorrhage in patients with untreated brain arteriovenous malformation. Neurology 2006;66:1350-55

6. Ogilvy CS, Stieg PE, Awad I, et al. Recommendations for the management of intracranial arteriovenous malformations: a statement for healthcare professionals from a special writing group of the Stroke Council, American Stroke Association. Circulation 2001; 103:2644-57

7. Andrews BT, Wilson CB. Staged treatment of arteriovenous malformations of the brain. Neurosurgery 1987;21:314-23

8. Unlu E, Temizoz O, Albayram S, et al. Contrast-enhanced MR 3D angiography in the assessment of brain AVMs. Eur J Radiol 2006;60:367-78

9. Hadizadeh DR, von Falkenhausen M, Gieseke J, et al. Cerebral arteriovenous malformation: Spetzler-Martin classification at subsecond-temporal-resolution four-dimensional MR angiography compared with that at DSA. Radiology 2008;246:205-13

10. Eddleman CS, Jeong HJ, Hurley MC, et al. 4D radial acquisition contrast-enhanced MR angiography and intracranial arteriovenous malformations: quickly approaching digital subtraction angiography. Stroke 2009;40:2749-53

11. Nishimura S, Hirai T, Sasao A, et al. Evaluation of dural arteriovenous fistulas with $4 \mathrm{D}$ contrast-enhanced MR angiography at 3T. AJNR Am J Neuroradiol 2010;31:80-85

12. Illies T, Forkert ND, Ries T, et al. Classification of cerebral arteriovenous malformations and intranidal flow patterns by color-encoded 4D-hybrid-MRA. AJNR Am J Neuroradiol 2013;34:46-53

13. Forkert ND, Fiehler J, Illies T, et al. 4D blood flow visualization fusing 3D and 4D MRA image sequences. J Magn Reson Imaging 2012;36:443-53

14. Bammer R, Hope TA, Aksoy M, et al. Time-resolved 3D quantitative flow MRI of the major intracranial vessels: initial experience and comparative evaluation at $1.5 \mathrm{~T}$ and $3.0 \mathrm{~T}$ in combination with parallel imaging. Magn Reson Med 2007;57:127-40

15. Wetzel S, Meckel S, Frydrychowicz A, et al. In vivo assessment and visualization of intracranial arterial hemodynamics with flow-sensitized 4D MR imaging at 3T. AJNR Am J Neuroradiol 2007;28:433-38

16. Hope TA, Hope MD, Purcell DD, et al. Evaluation of intracranial stenoses and aneurysms with accelerated 4D flow. Magn Reson Imaging 2010;28:41-46
17. Isoda $\mathrm{H}$, Ohkura $\mathrm{Y}$, Kosugi $\mathrm{T}$, et al. In vivo hemodynamic analysis of intracranial aneurysms obtained by magnetic resonance fluid dynamics (MRFD) based on time-resolved three-dimensional phasecontrast MRI. Neuroradiology 2010;52:921-28

18. Rayz VL, Boussel L, Ge L, et al. Flow residence time and regions of intraluminal thrombus deposition in intracranial aneurysms. Ann Biomed Eng 2010;38:3058-69

19. Hope MD, Purcell DD, Hope TA, et al. Complete intracranial arterial and venous blood flow evaluation with 4D flow MR imaging. AJNR Am J Neuroradiol 2009;30:362-66

20. Chang W, Loecher MW, Wu Y, et al. Hemodynamic changes in patients with arteriovenous malformations assessed using high-resolution $3 \mathrm{D}$ radial phase-contrast magnetic resonance angiography. AJNR Am J Neuroradiol 2012;33:1565-72

21. Meckel S, Stalder AF, Santini F, et al. In vivo visualization and analysis of 3-D hemodynamics in cerebral aneurysms with flow-sensitized 4-D MR imaging at 3 T. Neuroradiology 2008;50:473-84

22. Bock J, Kreher BW, Hennig J, et al. Optimized pre-processing of timeresolved 2D and 3D phase contrast MRI data. In Proceedings: $15 \mathrm{th} \mathrm{Sci-}$ entific Meeting of the International Society for Magnetic Resonance in Medicine 2007:3138

23. Bock J, Frydrychowicz A, Stalder AF, et al. 4D phase contrast MRI at $3 \mathrm{~T}$ : effect of standard and blood-pool contrast agents on SNR, PC-MRA, and blood flow visualization. Magn Reson Med 2010;63:330-38

24. Markl M, Harloff A, Bley TA, et al. Time-resolved 3D MR velocity mapping at 3T: improved navigator-gated assessment of vascular anatomy and blood flow. J Magn Reson Imaging 2007;25:824-31

25. Harloff A, Simon J, Brendecke S, et al. Complex plaques in the proximal descending aorta: an underestimated embolic source of stroke. Stroke 2010;41:1145-50

26. Markl M, Geiger J, Kilner PJ, et al. Time-resolved three-dimensional magnetic resonance velocity mapping of cardiovascular flow paths in volunteers and patients with Fontan circulation. Eur J Cardiothorac Surg 2011;39:206-12

27. Fera F, Bono F, Messina D, et al. Comparison of different MR venography techniques for detecting transverse sinus stenosis in idiopathic intracranial hypertension. J Neurol 2005;252:1021-25

28. Saatci I, Cekirge HS, Ciceri EF, et al. CT and MR imaging findings and their implications in the follow-up of patients with intracranial aneurysms treated with endosaccular occlusion with Onyx. AJNR Am J Neuroradiol 2003;24:567-78

29. da Costa L, Wallace MC, Ter Brugge KG, et al. The natural history and predictive features of hemorrhage from brain arteriovenous malformations. Stroke 2009;40:100-05

30. Anzalone N, Scomazzoni F, Strada L, et al. Intracranial vascular malformations. Eur Radiol 1998;8:685-90

31. See AP, Raza S, Tamargo RJ, et al. Stereotactic radiosurgery of cranial arteriovenous malformations and dural arteriovenous fistulas. Neurosurg Clin N Am 2012;23:133-46

32. Hamm KD, Klisch J, Surber G, et al. Special aspects of diagnostic imaging for radiosurgery of arteriovenous malformations. Neurosurgery 2008;62:A44-52

33. Lv X, Wu Z, Li Y, et al. Hemorrhage risk after partial endovascular NBCA and Onyx embolization for brain arteriovenous malformation. Neurol Res 2012;34:552-56

34. Lv X, Wu Z, Jiang C, et al. Complication risk of endovascular embolization for cerebral arteriovenous malformation. Eur J Radiol 2011;80:776-79

35. Ogilvy CS, Stieg PE, Awad I, et al. AHA scientific statement: recommendations for the management of intracranial arteriovenous malformations: a statement for healthcare professionals from a special writing group of the Stroke Council, American Stroke Association. Stroke 2001;32:1458-71

36. Hartmann A, Mast H, Mohr JP, et al. Determinants of staged endovascular and surgical treatment outcome of brain arteriovenous malformations. Stroke 2005;36:2431-35 